\title{
The Service Quality Evaluation of Agricultural E-Commerce Based on Interval-Valued Intuitionistic Fuzzy GRA Method
}

\author{
Haibin Zhang $\mathbb{D}^{1,2}$ and Lei Wang $\mathbb{i D}^{3}$ \\ ${ }^{1}$ College of Management, Suqian University, Suqian 223800, China \\ ${ }^{2}$ College of Economics and Management, Zhejiang Agriculture and Forest University, Hangzhou 311300, China \\ ${ }^{3}$ College of Economics and Management, Anhui Science and Technology University, Bengbu 233100, China
}

Correspondence should be addressed to Haibin Zhang; zhanghaibin18@zafu.edu.cn

Received 12 April 2021; Revised 27 September 2021; Accepted 14 November 2021; Published 7 January 2022

Academic Editor: Feng Feng

Copyright $\odot 2022$ Haibin Zhang and Lei Wang. This is an open access article distributed under the Creative Commons Attribution License, which permits unrestricted use, distribution, and reproduction in any medium, provided the original work is properly cited.

\begin{abstract}
The service quality evaluation of agricultural business-to-customer (B2C) e-commerce is viewed as a multiattribute group decision-making (MAGDM) activity. Thus, a useful MAGDM process is required. Based on the grey relational analysis (GRA) process and the interval-valued intuitionistic fuzzy set (IVIFS), this study defines an interval-valued intuitionistic fuzzy (IVIF) GRA process to depict the service quality of agricultural B2C e-commerce. This is important to agricultural B2C e-commerce because this industry increases rapidly and many new services are innovated. In this article, some necessary definitions related to IVIFSs are reviewed. Additionally, criteria weights are derived using the Criteria Importance Through Intercriteria Correlation method (CRITIC). Subsequently, the GRA method is extended to incorporate IVIFs to obtain a final service alternative. All alternatives can then be ranked, and the best service quality option can be identified and promulgated. Finally, a numerical example and some useful comparative studies are obtained. The analysis results show that the defined algorithm is effective for identifying the service qualities of agricultural B2C e-commerce, which provide a new assessment method for MAGDM.
\end{abstract}

\section{Introduction}

Because the process of making decisions is full of uncertainty and ambiguity [1-3], Zadeh [4] designed fuzzy sets (FSs) to deal with decision-making accuracy issues when binary criteria and alternatives were not available. Atanassov [5] built intuitionistic fuzzy sets (IFSs) to depict uncertain issues. Then, Gupta et al. [6] designed fuzzy mathematical entropy under IFSs.

He et al. [7] built power interaction information-fused operators under IFSs. Chen et al. [8] developed the IFTOPSIS (Technique for Order Preference by Similarity to an Ideal Solution) method using similarity measures. Those research studies extended intuitionistic fuzzy set theory significantly. Interval-valued intuitionistic fuzzy set is very important for fuzzy theory, which not only considers membership degree and non-membership degree but also considers hesitant situation. Jin et al. [9] defined two methods to cope with priority weights under IFSs. Liu et al. [10] devised intuitionistic fuzzy decision-fused operators along with BM and Dombi for real MAGDM. After that, subsequent research studies concentrated on the combination of traditional MAGDM methods and IFSs. The MABAC (multiattribute border approximation area comparison) has a systematic procedure with simple computation process and a sound logic that represents the rationale of human decision making. The IF-MABAC method with distance measures was innovated by Liang et al. [11]. Zhang and $\mathrm{He}$ [12] built geometric interaction information-fused operators under IFSs. Xu and Yager [13] defined weighted geometric operators under IVIFs (IVIFWG). This method can improve opinion leader's weight between experts' assessment. Krishankumar et al. [14] built IF-PROMETHEE (Preference Ranking Organization Method for Enrichment Evaluation). $\mathrm{Li}$ and $\mathrm{Wu}$ [15] defined cross-entropy distance under IFSs. Joshi and Kumar [16] defined an extended 
VIKOR (VlseKriterijumska Optimizacija I Kompromisno Resenje) method using IFSs. Phochanikorn and Tan [17] merged DEMATEL (Decision-Making Trial and Evaluation Laboratory) with ANP (Analytic Network Process) to obtain interdependencies and uncertainties under IFSs. Li et al. [18] defined grey-target real decision making under IFSs. This method employs the thought of gray systems, which provides an innovative idea for this topic. Kumar and Garg [19] defined the TOPSIS method using IVIFSs. Different from the GRA method, TOPSIS did not concentrate on correlation. Based on the extension of IFSs and IVIFSs, He et al. [20] defined the Pythagorean 2-tuple linguistic EDAS method. The CODAS (Combinative Distance-Based Assessment) method uses the Euclidean distance and taxicab distance to measure alternatives according to the negativeideal point.

GRA was initially defined by Deng [21] to cope with a real MAGDM. Compared with other real MAGDM methods, the GRA method can consider the shape similarity of every given alternative from PIS and NIS. Tan et al. [22] defined design alternatives as well as the GRA process combined with AHP. Malek et al. [23] built a revised hybrid GRA for green supply. Yazdani et al. [24] provided the QFD and the GRA methods in dealing with supply chain drivers. Furthermore, some significant research studies are demonstrated as follows. Chen [25] linked the IF-GRA method with the entropy-TOPSIS process to select a material supplier. To discern the carbon market, Zhu et al. [26] took advantage of the GRA process as well as EMD. Chiang [27] used the GRA for dependent MADM. Alptekin et al. [28] solved low-carbon development based on the GRA process. Kung and Wen [29] used the GRA process to solve the grey MADM. Lei et al. [30] defined a probabilistic linguistic GRA method. Wei and Lan [31] solved MADM under IVIFSs using the GRA method. However, that study had the following limitations: it could not solve the MAGDM problem with the GRA method under IVIFSs, and it could solve the MAGDM problem under IVIFSs with GRA with unknown weight information.

Unfortunately, in the existing literature, it is very difficult to determine the corresponding works of the GRA method with CRITIC through IVIFS information. The GRA method is often used in grey systems which are partially understood by researchers. The IVIFS method considers both membership and non-membership and can effectively deal with uncertainty. Theoretical ideas behind these two methods were used to tackle unclear problems. Thus, it is imperative to extend the GRA process to IVIFSs. The primary aim of this study is to address some real MAGDM issues efficiently using GRA and IVIFSs. Moreover, this study extends the GRA process to IVIFSs. However, the CRITIC method is employed to derive the given attribute's weight. Then, a certified application is utilized to certify the defined model, and several useful comparative studies are utilized to certify the advantages of the defined model.

The major contribution and novelty of the work can be outlined as follows:

(1) The GRA method is used to cope with the MAGDM issue under IVIFSs.

(2) The weights of attributes are derived objectively through the CRITIC method.

(3) A numerical example study for evaluating the service quality of agricultural $\mathrm{B} 2 \mathrm{C}$ e-commerce is given to show the defined approach.

(4) Some useful comparative studies are provided with the existing methods.

The remainder of this paper is organized as follows. Some necessary ideas for IVIFSs are given in Section 2. The GRA process for real MAGDM is revised with IVIFSs, and the calculation procedures are described in Section 3. A numerical example for evaluating the service quality of agricultural $\mathrm{B} 2 \mathrm{C}$ e-commerce is given, and a comparative analysis is presented in Section 4. Finally, we give our conclusions in Section 5.

\section{Preliminaries}

\subsection{IVIFSs}

Definition 1 (see [32]). The IVIFS based on $X$ is

$$
K=\left\{\left\langle x, \tilde{\mu}_{K}(x), \widetilde{v}_{K}(x)\right\rangle \mid \quad x \in X\right\},
$$

where $\widetilde{\mu}_{K}(x) \subset[0,1]$ is depicted as "membership of $K$ " and $\widetilde{v}_{K}(x) \subset[0,1]$ is depicted as "non-membership of $K$," and $\tilde{\mu}_{K}(x), \quad \tilde{v}_{K}(x)$ could meet real condition: $0 \leq \sup \widetilde{\mu}_{K}(x)+\sup \widetilde{v}_{K}(x) \leq 1, \forall x \in X$.

Definition 2 (see [33]). Let $K_{1}=\left(\left[\mu_{1}^{L}, \mu_{1}^{R}\right],\left[\nu_{1}^{L}, v_{1}^{R}\right]\right)$ and $K_{2}=\left(\left[\mu_{2}^{L}, \mu_{2}^{R}\right],\left[\nu_{2}^{L}, v_{2}^{R}\right]\right)$ be two IVIFs; then, the operation rules between them are

$$
\begin{aligned}
K_{1} \oplus K_{2} & =\left(\left[\mu_{1}^{L}+\mu_{2}^{L}-\mu_{1}^{L} \mu_{2}^{L}, \mu_{1}^{R}+\mu_{2}^{R}-\mu_{1}^{R} \mu_{2}^{R}\right],\left[v_{1}^{L} v_{2}^{L}, v_{1}^{R} v_{2}^{R}\right]\right), \\
K_{1} \otimes K_{2} & =\left(\left[\mu_{1}^{L} \mu_{2}^{L}, \mu_{1}^{R} \mu_{2}^{R}\right],\left[v_{1}^{L}+\nu_{2}^{L}-v_{1}^{L} v_{2}^{L}, v_{1}^{R}+v_{2}^{R}-v_{1}^{R} v_{2}^{R}\right]\right), \\
\lambda K_{1} & =\left(\left[1-\left(1-\mu_{1}^{L}\right)^{\lambda}, 1-\left(1-\mu_{1}^{R}\right)^{\lambda}\right],\left[\left(v_{1}^{L}\right)^{\lambda},\left(v_{1}^{R}\right)^{\lambda}\right]\right), \quad \lambda>0, \\
K_{1}^{\lambda} & =\left(\left[\left(\mu_{1}^{L}\right)^{\lambda},\left(\mu_{1}^{R}\right)^{\lambda}\right],\left[1-\left(1-\lambda_{1}^{L}\right)^{\lambda}, 1-\left(1-\lambda_{1}^{R}\right)^{\lambda}\right]\right), \quad \lambda>0 .
\end{aligned}
$$


Definition 3 (see [34]). Let $S(K)=\left(\left[\mu^{L}, \mu^{R}\right],\left[v^{L}, v^{R}\right]\right)$ be given IVIFNs, and the score and accuracy values are defined as follows:

$$
\begin{aligned}
& S(K)=\frac{\left(\mu^{L}-v^{L}\right)+\left(\mu^{R}-v^{R}\right)}{2}, \\
& H(K)=\frac{\mu^{L}+v^{L}+\mu^{R}+v^{R}}{2} .
\end{aligned}
$$

For two given IVIFNs $K_{1}$ and $K_{2}$, from Definition 3,
(1) if $s\left(K_{1}\right)<s\left(K_{2}\right)$, then $K_{1}<K_{2}$;

(2) if $s\left(K_{1}\right)=s\left(K_{2}\right), h\left(K_{1}\right)<h\left(K_{2}\right), \quad$ then $K_{1}<K_{2}$;

(3) if $s\left(K_{1}\right)=s\left(K_{2}\right), h\left(K_{1}\right)=h\left(K_{2}\right)$, then $K_{1}=K_{2}$.

Definition 4 (see [35]). Let $K_{1}=\left(\left[\mu_{1}^{L}, \mu_{1}^{R}\right],\left[\nu_{1}^{L}, \nu_{1}^{R}\right]\right)$ and $K_{2}=\left(\left[\mu_{2}^{L}, \mu_{2}^{R}\right],\left[\nu_{2}^{L}, v_{2}^{R}\right]\right)$ be given IVIFNs; the Euclidean distance between two given IVIFNs is defined as

$$
\operatorname{ED}\left(K_{1}, K_{2}\right)=\sqrt{\frac{1}{4}\left[\left(\mu_{1}^{L}-\mu_{2}^{L}\right)^{2}+\left(\mu_{1}^{R}-\mu_{2}^{R}\right)^{2}+\left(v_{1}^{L}-v_{2}^{L}\right)^{2}+\left(v_{1}^{R}-v_{2}^{R}\right)^{2}\right]}
$$

2.2. Two Operators under IVIFSs. Under the setting of the IVIFSs, some operators could be reviewed, including IVIFWA operator and IVIFWG operator [36].
Definition 5 (see [36]). Let $K_{j}=\left(\left[\mu_{K_{j}}^{L}, \mu_{K_{j}}^{R}\right],\left[v_{K_{j}}^{L}, v_{K_{j}}^{R}\right]\right)(j=$ $1,2, \ldots, n)$ be a set of given IVIFNs, and the given IVIFWA operator is

$$
\begin{aligned}
\operatorname{IVIFWA}_{\omega}\left(K_{1}, K_{2}, \ldots, K_{n}\right) & =\underset{j=1}{\oplus}\left(\omega_{j} K_{j}\right) \\
& =\left(\left[1-\prod_{j=1}^{n}\left(1-\mu_{K_{j}}^{L}\right)^{\omega_{j}}, 1-\prod_{j=1}^{n}\left(1-\mu_{K_{j}}^{R}\right)^{\omega_{j}}\right],\left[\prod_{j=1}^{n}\left(v_{K_{j}}^{L}\right)^{\omega_{j}}, \prod_{j=1}^{n}\left(v_{K_{j}}^{R}\right)^{\omega_{j}}\right]\right),
\end{aligned}
$$

where $\omega=\left(\omega_{1}, \omega_{2}, \ldots, \omega_{n}\right)^{T}$ is the weight of Definition 6 (see [37]). Let $K_{j}=\left(\left[\mu_{K_{j}}^{L}, \mu_{K_{j}}^{R}\right]\right.$, [ $\left.\left.\nu_{K_{j}}^{L}, v_{K_{j}}^{R}\right]\right)(j=$ $K_{j}(j=1,2, \ldots, n)$ and $\omega_{j}>0, \sum_{j=1}^{n} \omega_{j}=1$. $1,2, \ldots, n)$ be a set of given IVIFNs, and the given IVIFWG operator is

$$
\begin{aligned}
\operatorname{IVIFWG}_{\omega}\left(K_{1}, K_{2}, \ldots, K_{n}\right) & =\stackrel{\otimes}{j=1}_{\left(K_{j}\right)^{\omega_{j}}} \\
& =\left(\left[\prod_{j=1}^{n}\left(\mu_{K_{j}}^{L}\right)^{\omega_{j}}, \prod_{j=1}^{n}\left(\mu_{K_{j}}^{R}\right)^{\omega_{j}}\right],\left[1-\prod_{j=1}^{n}\left(1-v_{K_{j}}^{L}\right)^{\omega_{j}}, 1-\prod_{j=1}^{n}\left(1-v_{K_{j}}^{R}\right)^{\omega_{j}}\right]\right),
\end{aligned}
$$

where $\omega=\left(\omega_{1}, \omega_{2}, \ldots, \omega_{n}\right)^{T}$ is the weight of $K_{j}(j=1,2, \ldots, n)$ and $\omega_{j}>0, \sum_{j=1}^{n} \omega_{j}=1$.

\section{GRA Process for MAGDM with IVIFNs}

This section builds the IVIF-GRA process for defined real MAGDM. The designed calculating steps of defined method could be always depicted. Let $T A=\left\{T A_{1}, T A_{2}, \ldots T A_{n}\right\}$ be the set of attributes and $t a=\left\{t a_{1}, t a_{2}, \ldots t a_{n}\right\}$ be the given attribute weight $T A_{j}$, where $t a_{j} \in[0,1], j=1,2, \ldots$, $n, \sum_{j=1}^{n} t a_{j}=1$. Let $H A=\left\{H A_{1}, H A_{2}, \ldots H A_{l}\right\}$ be a group of DMs that have remarkable degree of $h a=\left\{h a_{1}, h a_{2}, \ldots h a_{l}\right\}$, where $h a_{k} \in[0,1], k=1,2, \ldots, l$. $\sum_{k=1}^{l} h a_{k}=1$. Let $P A=\left\{P A_{1}, P A_{2}, \ldots P A_{m}\right\}$ be a group of given alternatives. $Q A=\left(q a_{i j}\right)_{m \times n}$ is the decision matrix, and $q a_{i j}$ depicts the value of $P A_{i}$ for $T A_{j}$. Afterwards, the given calculating steps are listed.

Step 1. Build the matrix $Q A^{(k)}=\left(q a_{i j}^{k}\right)_{m \times n}$; then, derive the overall matrix $Q A=\left(q a_{i j}\right)_{m \times n}$. 


$$
\begin{aligned}
Q A^{(k)}=\left[q a_{i j}^{k}\right]_{m \times n}= & {\left[\begin{array}{cccc}
q a_{11}^{k} & q a_{12}^{k} & \cdots & q a_{1 n}^{k} \\
q a_{21}^{k} & q a_{22}^{k} & \cdots & q a_{2 n}^{k} \\
\vdots & \vdots & \vdots & \vdots \\
q a_{m 1}^{k} & q a_{m 2}^{k} & \cdots & q a_{m n}^{k}
\end{array}\right], \quad k(k=1,2, \ldots, k), } \\
Q A & =\left[q a_{i j}\right]_{m \times n}=\left[\begin{array}{cccc}
q a_{11} & q a_{12} & \cdots & q a_{1 n} \\
q a_{21} & q a_{22} & \cdots & q a_{2 n} \\
\vdots & \vdots & \vdots & \vdots \\
q a_{m 1} & q a_{m 2} & \cdots & q a_{m n}
\end{array}\right], \\
q a_{i j} & =\left(\left[1-\prod_{k=1}^{l}\left(1-\mu_{q a_{i j}^{k}}^{L}\right)^{h a_{k}}, 1-\prod_{k=1}^{l}\left(1-\mu_{q a_{i j}^{k}}^{R}\right)^{h a_{k}}\right],\left[\prod_{k=1}^{l}\left(v_{q a_{i j}^{k}}^{L}\right)^{h a_{k}}, \prod_{k=1}^{l}\left(v_{q a_{i j}^{k}}^{R}\right)^{h a_{k}}\right]\right),
\end{aligned}
$$

where $q a_{i j}^{k}$ is the assessment information of $P A_{i}(i=$ $1,2, \ldots, m)$ for $T A_{j}(j=1,2, \ldots, n) \quad$ and $H A_{k}(k=1,2, \ldots, l)$.

Step 2. Normalize the mathematical matrix $\mathrm{Q} A=\left(q a_{i j}\right)_{m \times n}$ to $\mathrm{Q} A^{N}=\left[q a_{i j}^{N}\right]_{m \times n}$ with IVIFNs.

$q a_{i j}^{N}= \begin{cases}\left(\left[\mu_{i j}^{L}, \mu_{i j}^{R}\right],\left[v_{i j}^{L}, v_{i j}^{R}\right]\right), & T A_{j} \text { is a benefit criterion, } \\ \left(\left[v_{i j}^{L}, v_{i j}^{R}\right],\left[\mu_{i j}^{L}, \mu_{i j}^{R}\right]\right), & T A_{j} \text { is a cost criterion. }\end{cases}$
Step 3. Take advantage of the CRITIC to obtain the attributes' weight.

The CRITIC process was defined to decide attributes' weights. This method was defined by Diakoulaki et al. [38] which took the correlations relationship between given attributes into consideration, whereafter the computing procedures of such method are designed.

Depending on the normalized matrix, $Q^{N}=\left(q a_{i j}^{n}\right)_{m \times n}$, the correlation coefficient between attributes could be obtained.

$$
\mathrm{CC}_{j r}=\frac{\sum_{i=1}^{m}\left(S\left(q a_{i j}^{N}\right)-S\left(q a_{j}^{N}\right)\right)\left(S\left(q a_{i r}^{N}\right)-S\left(q a_{r}^{N}\right)\right)}{\sqrt{\sum_{i=1}^{m}\left(S\left(q a_{i j}^{N}\right)-S\left(q a_{j}^{N}\right)\right)^{2}} \sqrt{\sum_{i=1}^{m}\left(S\left(q a_{i r}^{N}\right)-S\left(q a_{r}^{N}\right)\right)^{2}}}, \quad j, r=1,2, \ldots, n
$$

where $\quad S\left(q a_{j}^{N}\right)=(1 / m) \sum_{i=1}^{m} S\left(q a_{i j}^{N}\right) \quad$ and $S\left(q a_{r}^{N}\right)=(1 / m) \sum_{i=1}^{m} S\left(q a_{i r}^{N}\right)$.

Calculate attributes' standard deviation.

$\mathrm{SD}_{j}=\sqrt{\frac{1}{m-1} \sum_{i=1}^{m}\left(S\left(q a_{i j}^{N}\right)-S\left(q a_{j}^{N}\right)\right)^{2}}, \quad j=1,2, \ldots, n$,

where $S\left(q_{j}^{N}\right)=(1 / m) \sum_{i=1}^{m} S\left(q_{i j}^{N}\right)$.

Calculate the attributes' weights.

$$
t a_{j}=\frac{\mathrm{SD}_{j} \sum_{t=1}^{n}\left(1-\mathrm{CC}_{j r}\right)}{\sum_{j=1}^{n}\left(\mathrm{SD}_{j} \sum_{t=1}^{n}\left(1-\mathrm{CC}_{j r}\right)\right)}, \quad j=1,2 \ldots, n,
$$

where $t a_{j} \in[0,1]$ and $\sum_{j=1}^{n} t a_{j}=1$.
Step 4. Build positive ideal solutions (PISs) IVIFPIS $_{j}$ and the corresponding negative ideal solutions (NISs) IVIFNIS $_{j}$ through equations (15) and (16):

$$
\begin{gathered}
\text { IVIFPIS }_{j}=\left(\left[\mu_{j}^{L+}, \mu_{j}^{R+}\right],\left[v_{j}^{L+}, v_{j}^{R+}\right]\right), \\
\text { IVIFNIS }_{j}=\left(\left[\mu_{j}^{L+}, \mu_{j}^{R+}\right],\left[v_{j}^{L+}, v_{j}^{R+}\right]\right), \\
\text { where } \text { IVIFPIS }_{j}=\left(\begin{array}{c}
{\left[\max _{j}\left(\mu_{i j}^{L}\right), \max _{j}\left(\mu_{i j}^{R}\right)\right],} \\
{\left[\min _{j}\left(v_{i j}^{L}\right), \min _{j}\left(v_{i j}^{R}\right)\right]}
\end{array}\right) \text { and } \\
\text { IVIFNIS }_{j}=\left(\begin{array}{c}
{\left[\min _{j}\left(\mu_{i j}^{L}\right), \min _{j}\left(\mu_{i j}^{R}\right)\right],} \\
{\left[\max _{j}\left(v_{i j}^{L}\right), \max _{j}\left(v_{i j}^{R}\right)\right]}
\end{array}\right) .
\end{gathered}
$$

Step 5. The important point of computing grey rational coefficients emphasizes shape similarity degree of every given attribute of every given alternative from the IVIFPIS and IVIFNIS. Thus, compute the grey relational coefficient (GRC) of each given alternative 
between each given alternative and IVIF-IVPIS and the GRC between each given alternative and IVIF-PIS as

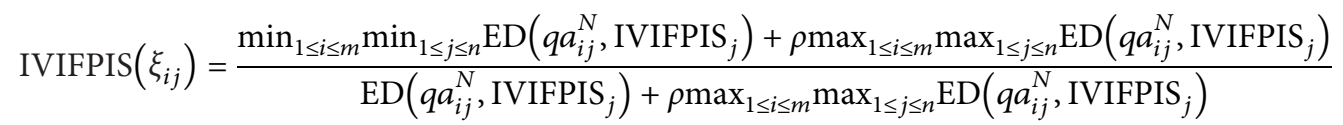

$$
\begin{aligned}
& \operatorname{IVIFNIS}\left(\xi_{i j}\right)=\frac{\min _{1 \leq i \leq m} \min _{1 \leq j \leq n} \operatorname{ED}\left(q a_{i j}^{N}, \text { IVIFPIS }_{j}\right)+\rho \max _{1 \leq i \leq m} \max _{1 \leq j \leq n} \operatorname{ED}\left(q a_{i j}^{N}, \text { IVIFPIS }_{j}\right)}{\operatorname{ED}\left(q a_{i j}^{N}, \operatorname{IVIFNIS}{ }_{j}\right)+\rho \max _{1 \leq i \leq m} \max _{1 \leq j \leq n} \operatorname{ED}\left(q a_{i j}^{N}, \operatorname{IVIFNIS}_{j}\right)}, \\
& i=1,2, \ldots, m, j=1,2, \ldots, n .
\end{aligned}
$$

Step 6. Figure out the degree of GRC of all given alternatives from IVIFPIS as well as IVIFNIS:

$$
\begin{aligned}
& \operatorname{IVIFPIS}\left(\xi_{i}\right)=\sum_{j=1}^{n} t a_{j} \operatorname{IVIFPIS}\left(\xi_{i j}\right), \quad i=1,2, \ldots, m, \\
& \operatorname{IVIFNIS}\left(\xi_{i}\right)=\sum_{j=1}^{n} t a_{j} \operatorname{IVIFNIS}\left(\xi_{i j}\right), \quad i=1,2, \ldots, m .
\end{aligned}
$$

The basic idea of the GRA process is that the given alternative could have the "largest degree of grey relation" from the IVIFPIS and the "smallest degree of grey relation" from the IVIFNIS.

Step 7. Compute each alternative's interval-valued intuitionistic fuzzy relative relational degree (IVIFRRD) of all given alternatives from IVIFPIS:

$$
\text { IVIFRRD }_{i}
$$

$$
=\frac{\operatorname{IVIFNIS}\left(\xi_{i}\right)}{\operatorname{IVIFNIS}\left(\xi_{i}\right)+\operatorname{IVIFPIS}\left(\xi_{i}\right)}, \quad i=1,2, \ldots, m .
$$

Step 8. The optimal choice can be determined by the highest value of IVIFRRD.

\section{Illustrative Example and Some Comparative Analyses}

4.1. Numerical Example. In the last few years, Internet shopping has been developing rapidly in China, exerting a great influence on traditional pure offline formats such as department stores, supermarkets, and physical fresh stores. According to the data released by China's agricultural fresh e-commerce development BBS in 2016, more than $80 \%$ of traditional fresh e-commerce enterprises failed to make profits. The pure online Internet shopping model can no longer satisfy the needs of Chinese consumers. The new retail model of "online + offline + strong logistics + big data" has become the favorite of the new era. As an emerging business model, the new retail model is based on the Internet, big data technology, and intelligent logistics to effectively organize and integrate online and offline data and resources. Gronroos [39] defined service quality as "a kind of perception, which is determined by the comparison between customer's service expectation and actual service experience." There are two classical theory frameworks for service quality measurement. Parasuraman et al. [40] proposed the SERVQUAL (service quality) model for service quality evaluation, which is also named the PZB model. The other one is SERVPERF (service performance) which was proposed by Cronin and Taylor [41]. Compared with SERVQUAL, SERVPERF is easier for investigation because it supports one-shot measurement. However, this study did not aim to develop or extend this famous theory; this framework was used to develop a new methodology. Thus, a few service research papers were reviewed. Three attributes were borrowed directly from SERVQUAL. Furthermore, "customer complaint" was the fourth attribute to represent cost attribute in the research. In this part, an empirical application is given to evaluate to the service quality of agricultural B2C e-commerce, which could be solved through IVIF-GRA model. Since the local government hopes to choose the urban road through the best environmental behaviors, there are five latent enterprises $\mathrm{EP}_{i}(i=1,2,3,4,5)$. For evaluating the service quality evaluation of agricultural $\mathrm{B} 2 \mathrm{C}$ e-commerce fairly, five experts $\mathrm{EH}=\left\{\mathrm{EH}_{1}, \mathrm{EH}_{2}, \mathrm{EH}_{3}, \mathrm{EH}_{4}, \mathrm{EH}_{5}\right\}$ (expert's weight ew $=(0.2,0.2,0.2,0.2)$ are asked. All invited experts express their assessed information through four attributes: (1) $\mathrm{ET}_{1}$ is assurance of agriculture e-commerce; (2) $\mathrm{ET}_{2}$ is customer complaint of agriculture e-commerce; (3) $\mathrm{ET}_{3}$ is reliability of e-commerce; and (4) $\mathrm{ET}_{4}$ is responsiveness of e-commerce. Evidently, $\mathrm{ET}_{2}$ is cost attribute, while $\mathrm{ET}_{1}, \mathrm{ET}_{3}$, and $\mathrm{ET}_{4}$ are given benefit attributes.

(i) Step 1. Build each given IVIF matrix $Q A^{(k)}=\left(q a_{i j}^{k}\right)_{m \times n}$ as in Tables 1-5. From the tables and equations (8)-(10), the overall matrix shall be obtained. The calculating results are defined in Tables 1-6.

(ii) Step 2. Normalize the IVIF information matrix $E Q=\left[q a_{i j}\right]_{m \times n}$ to $E Q^{N}=\left[q a_{i j}^{N}\right]_{m \times n}$ (see Table 7).

(iii) Step 3. The weights can be calculated $t a_{j}(j=1,2, \ldots, n)$ through CRITIC (see Table 8). 
TABLE 1: IVIF matrix by $H_{1}$.

\begin{tabular}{lcccc}
\hline & $\mathrm{ET}_{1}$ & $\mathrm{ET}_{2}$ & $\mathrm{ET}_{3}$ & $\mathrm{ET}_{4}$ \\
\hline $\mathrm{EP}_{1}$ & $([0.55,0.62],[0.27,0.38])$ & $([0.29,0.35],[0.60,0.65])$ & $([0.32,0.40],[0.55,0.60])$ & $([0.35,0.40],[0.51,0.60])$ \\
$\mathrm{EP}_{2}$ & $([0.28,0.46],[0.50,0.54])$ & $([0.53,0.60],[0.35,0.40])$ & $([0.60,0.65],[0.30,0.35])$ & $([0.62,0.70],[0.25,0.30])$ \\
$\mathrm{EP}_{3}$ & $([0.52,0.60],[0.35,0.40])$ & $([0.46,0.52],[0.40,0.48])$ & $([0.47,0.55],[0.40,0.45])$ & $([0.25,0.31],[0.66,0.71])$ \\
$\mathrm{EP}_{4}$ & $([0.36,0.41],[0.56,0.59])$ & $([0.41,0.45],[0.50,0.55])$ & $([0.60,0.70],[0.25,0.30])$ & $([0.73,0.81],[0.14,0.22])$ \\
$\mathrm{EP}_{5}$ & $([0.70,0.80],[0.15,0.20])$ & $([0.36,0.40],[0.57,0.60])$ & $([0.29,0.36],[0.58,0.64])$ & $([0.57,0.62],[0.30,0.38])$ \\
\hline
\end{tabular}

TABLE 2: IVIF matrix by $H_{2}$.

\begin{tabular}{lcccc}
\hline & $\mathrm{ET}_{1}$ & $\mathrm{ET}_{2}$ & $\mathrm{ET}_{3}$ & $\mathrm{ET}_{4}$ \\
\hline $\mathrm{EP}_{1}$ & $([0.38,0.42],[0.52,0.58])$ & $([0.61,0.65],[0.30,0.35])$ & $([0.37,0.45],[0.50,0.55])$ & $([0.55,0.60],[0.32,0.40])$ \\
$\mathrm{EP}_{2}$ & $([0.61,0.65],[0.30,0.35])$ & $([0.35,0.45],[0.50,0.55])$ & $([0.70,0.80],[0.10,0.20])$ & $([0.52,0.62],[0.30,0.38])$ \\
$\mathrm{EP}_{3}$ & $([0.63,0.70],[0.25,0.30])$ & $([0.59,0.62],[0.26,0.38])$ & $([0.19,0.25],[0.70,0.75])$ & $([0.59,0.65],[0.30,0.35])$ \\
$\mathrm{EP}_{4}$ & $([0.35,0.40],[0.55,0.60])$ & $([0.65,0.75],[0.20,0.25])$ & $([0.62,0.70],[0.25,0.30])$ & $([0.37,0.45],[0.55,0.60])$ \\
$\mathrm{EP}_{5}$ & $([0.42,0.48],[0.50,0.52])$ & $([0.37,0.40],[0.53,0.60])$ & $([0.36,0.40],[0.55,0.60])$ & $([0.55,0.62],[0.28,0.38])$ \\
\hline
\end{tabular}

TABLE 3: IVIF matrix by $H_{3}$.

\begin{tabular}{lcccc}
\hline & $\mathrm{ET}_{1}$ & $\mathrm{ET}_{2}$ & $\mathrm{ET}_{3}$ & $\mathrm{ET}_{4}$ \\
\hline $\mathrm{EP}_{1}$ & $([0.28,0.36],[0.57,0.64])$ & $([0.32,0.39],[0.41,0.61])$ & $([0.35,0.40],[0.55,0.60])$ & $([0.68,0.75],[0.20,0.25])$ \\
$\mathrm{EP}_{2}$ & $([0.44,0.48],[0.50,0.52])$ & $([0.25,0.30],[0.55,0.70])$ & $([0.58,0.63],[0.30,0.37])$ & $([0.41,0.52],[0.40,0.48])$ \\
$\mathrm{EP}_{3}$ & $([0.34,0.42],[0.50,0.58])$ & $([0.17,0.22],[0.65,0.78])$ & $([0.52,0.62],[0.32,0.38])$ & $([0.74,0.80],[0.15,0.21])$ \\
$\mathrm{EP}_{4}$ & $([0.18,0.25],[0.70,0.75])$ & $([0.32,0.40],[0.55,0.60])$ & $([0.66,0.75],[0.20,0.25])$ & $([0.59,0.66],[0.30,0.35])$ \\
$\mathrm{EP}_{5}$ & $([0.32,0.40],[0.55,0.60])$ & $([0.43,0.47],[0.50,0.53])$ & $([0.60,0.65],[0.30,0.35])$ & $([0.57,0.62],[0.32,0.38])$ \\
\hline
\end{tabular}

TABLE 4: IVIF matrix by $H_{4}$.

\begin{tabular}{lcccc}
\hline & $\mathrm{ET}_{1}$ & $\mathrm{ET}_{2}$ & $\mathrm{ET}_{3}$ & $\mathrm{ET}_{4}$ \\
\hline $\mathrm{EP}_{1}$ & $([0.36,0.42],[0.52,0.59])$ & $([0.56,0.62],[0.30,0.38])$ & $([0.31,0.42],[0.50,0.58])$ & $([0.71,0.78],[0.17,0.22])$ \\
$\mathrm{EP}_{2}$ & $([0.59,0.65],[0.30,0.35])$ & $([0.52,0.60],[0.35,0.41])$ & $([0.19,0.30],[0.65,0.70])$ & $([0.23,0.34],[0.58,0.66])$ \\
$\mathrm{EP}_{3}$ & $([0.38,0.45],[0.50,0.55])$ & $([0.72,0.80],[0.15,0.20])$ & $([0.73,0.80],[0.10,0.20])$ & $([0.60,0.66],[0.30,0.34])$ \\
$\mathrm{EP}_{4}$ & $([0.63,0.75],[0.15,0.25])$ & $([0.65,0.72],[0.21,0.28])$ & $([0.55,0.60],[0.30,0.40])$ & $([0.50,0.50],[0.50,0.50])$ \\
$\mathrm{EP}_{5}$ & $([0.55,0.60],[0.30,0.40])$ & $([0.65,0.70],[0.25,0.30])$ & $([0.24,0.35],[0.58,0.65])$ & $([0.43,0.46],[0.51,0.54])$ \\
\hline
\end{tabular}

TABLE 5: IVIF matrix by $H_{5}$.

\begin{tabular}{lcccc}
\hline & $\mathrm{ET}_{1}$ & $\mathrm{ET}_{2}$ & $\mathrm{ET}_{3}$ & $\mathrm{ET}_{4}$ \\
\hline $\mathrm{EP}_{1}$ & $([0.59,0.65],[0.27,0.35])$ & $([0.33,0.43],[0.51,0.57])$ & $([0.26,0.32],[0.60,0.68])$ & $([0.19,0.25],[0.19,0.25])$ \\
$\mathrm{EP}_{2}$ & $([0.40,0.45],[0.50,0.55])$ & $([0.41,0.45],[0.50,0.55])$ & $([0.34,0.41],[0.52,0.59])$ & $([0.33,0.42],[0.50,0.58])$ \\
$\mathrm{EP}_{3}$ & $([0.46,0.55],[0.30,0.45])$ & $([0.35,0.40],[0.50,0.60])$ & $([0.57,0.67],[0.26,0.33])$ & $([0.28,0.35],[0.58,0.65])$ \\
$\mathrm{EP}_{4}$ & $([0.34,0.41],[0.53,0.59])$ & $([0.28,0.32],[0.60,0.68])$ & $([0.69,0.75],[0.18,0.25])$ & $([0.41,0.55],[0.37,0.45])$ \\
$\mathrm{EP}_{5}$ & $([0.27,0.34],[0.60,0.66])$ & $([0.49,0.55],[0.40,0.45])$ & $([0.39,0.45],[0.48,0.55])$ & $([0.62,0.72],[0.20,0.28])$ \\
\hline
\end{tabular}

TABLE 6: Overall IVIF evaluation matrix.

\begin{tabular}{lcccc}
\hline & $\mathrm{ET}_{1}$ & $\mathrm{ET}_{2}$ & $\mathrm{ET}_{3}$ & $\mathrm{ET}_{4}$ \\
\hline $\mathrm{EP}_{1}$ & $([0.432,0.494],[0.432,0.508])$ & $([0.422,0.468],[0.424,0.512])$ & $([0.322,0.398],[0.540,0.602])$ & $([0.496,0.556],[0.278,0.344])$ \\
$\mathrm{EP}_{2}$ & $([0.464,0.538],[0.420,0.462])$ & $([0.412,0.480],[0.450,0.522])$ & $([0.482,0.558],[0.374,0.442])$ & $([0.422,0.520],[0.406,0.480])$ \\
$\mathrm{EP}_{3}$ & $([0.466,0.544],[0.380,0.456])$ & $([0.458,0.512],[0.392,0.488])$ & $([0.402,0.578],[0.364,0.422])$ & $([0.492,0.554],[0.398,0.452])$ \\
$\mathrm{EP}_{4}$ & $([0.372,0.444],[0.498,0.616])$ & $([0.462,0.528],[0.412,0.472])$ & $([0.624,0.700],[0.236,0.300])$ & $([0.520,0.594],[0.372,0.424])$ \\
$\mathrm{EP}_{5}$ & $([0.452,0.474],[0.420,0.476])$ & $([0.460,0.504],[0.450,0.496])$ & $([0.376,0.442],[0.498,0.558])$ & $([0.548,0.608],[0.322,0.392])$ \\
\hline
\end{tabular}


TABLE 7: The normalized IVIF matrix.

\begin{tabular}{lcccc}
\hline & $\mathrm{ET}_{1}$ & $\mathrm{ET}_{2}$ & $\mathrm{ET}_{3}$ & $\mathrm{ET}_{4}$ \\
\hline $\mathrm{EP}_{1}$ & $([0.432,0.494],[0.432,0.508])$ & $([0.424,0.512],[0.422,0.468])$ & $([0.322,0.398],[0.540,0.602])$ & $([0.496,0.556],[0.278,0.344])$ \\
$\mathrm{EP}_{2}$ & $([0.464,0.538],[0.420,0.462])$ & $([0.450,0.522],[0.412,0.480])$ & $([0.482,0.558],[0.374,0.442])$ & $([0.422,0.520],[0.406,0.480])$ \\
$\mathrm{EP}_{3}$ & $([0.466,0.544],[0.380,0.456])$ & $([0.392,0.488],[0.458,0.512])$ & $([0.402,0.578],[0.364,0.422])$ & $([0.492,0.554],[0.398,0.452])$ \\
$\mathrm{EP}_{4}$ & $([0.372,0.444],[0.498,0.616])$ & $([0.412,0.472],[0.462,0.528])$ & $([0.624,0.700],[0.236,0.300])$ & $([0.520,0.594],[0.372,0.424])$ \\
$\mathrm{EP}_{5}$ & $([0.452,0.474],[0.420,0.476])$ & $([0.450,0.496],[0.460,0.504])$ & $([0.376,0.442],[0.498,0.558])$ & $([0.548,0.608],[0.322,0.392])$ \\
\hline
\end{tabular}

TABLE 8: The weights $t a_{j}$.

\begin{tabular}{ccccc}
\hline & ET1 & ET2 & ET3 & ET4 \\
\hline$t a_{j}$ & 0.3368 & 0.1733 & 0.1247 & 0.3562 \\
\hline
\end{tabular}

TABLE 9: GRC of each alternative from IVIFPIS.

\begin{tabular}{lcccr}
\hline Alternatives & $\mathrm{ET}_{1}$ & $\mathrm{ET}_{2}$ & $\mathrm{ET}_{3}$ & \\
\hline $\mathrm{EP}_{1}$ & 0.6825 & 1.0000 & 0.3333 & \\
$\mathrm{EP}_{2}$ & 0.5443 & 0.4343 & 0.4175 & 0.4095 \\
$\mathrm{EP}_{3}$ & 0.7049 & 0.6825 & 1.0000 & 0.4943 \\
$\mathrm{EP}_{4}$ & 0.5181 & 0.4943 & 0.4343 & 1.0000 \\
$\mathrm{EP}_{5}$ & 1.0000 & 0.4343 & 0.4175 & 0.4831 \\
\hline
\end{tabular}

TABLE 10: GRC of each alternative from IVIFNIS.

\begin{tabular}{lcccc}
\hline Alternatives & $\mathrm{ET}_{1}$ & $\mathrm{ET}_{2}$ & $\mathrm{ET}_{3}$ & \\
\hline $\mathrm{EP}_{1}$ & 0.5172 & 0.3488 & 0.4167 & \\
$\mathrm{EP}_{2}$ & 0.7895 & 1.0000 & 1.0000 & 0.4545 \\
$\mathrm{EP}_{3}$ & 0.4412 & 0.3659 & 0.3333 & 0.8333 \\
$\mathrm{EP}_{4}$ & 1.0000 & 0.5172 & 0.7143 & 0.3947 \\
$\mathrm{EP}_{5}$ & 0.4286 & 0.4545 & 0.4839 & 1.0000 \\
\hline
\end{tabular}

(iv) Step 4. Determine the IVIFPIS IV $_{j}$ and IVIFNIS $_{j}$ by using equations (15) and (16).

$$
\begin{aligned}
& \text { IVIFPIS }_{1}=\langle(0.5876,0.6829),(0.2512,0.3172)\rangle, \\
& \text { IVIFPIS }_{2}=\langle(0.4522,0.4195),(0.5082,0.5805)\rangle, \\
& \text { IVIFPIS }_{3}=\langle(0.4703,0.5187),(0.3848,0.4723)\rangle, \\
& \text { IVIFPIS }_{4}=\langle(0.5627,0.6405),(0.2908,0.3171) 593\rangle, \\
& \text { IVIFNIS }_{1}=\langle(0.3011,0.3678),(0.5678,0.6323)\rangle, \\
& \text { IVIFNIS }_{2}=\langle(0.1661,0.2502),(0.6574,0.7498)\rangle, \\
& \text { IVIFNIS }_{3}=\langle(0.3036,0.3589),(0.5872,0.6411)\rangle, \\
& \text { IVIFNIS }_{4}=\langle(0.3162,0.4103),(0.5152,0.5892)\rangle .
\end{aligned}
$$

(v) Step 5. Figure out the GRC of every alternative from IVIFPIS as well as IVIFNIS (Tables 9 and 10).

(vi) Step 6. Figure out the degree of GRC of all alternatives from IVIFPIS as well as IVIFNIS (Table 11).

(vii) Step 7. Calculate the $\operatorname{IVIFRRD}\left(\xi_{i}\right)$ of each given alternative from IVIFPIS (Table 12).

According to the $\operatorname{IVIFRRD}\left(\xi_{i}\right)$, all given alternatives are ranked, the higher $\operatorname{IVIFRRD}\left(\xi_{i}\right)$, the better the alternative selected. Evidently, the order is $\mathrm{EP}_{3}>\mathrm{EP}_{1}>\mathrm{EP}_{5}>\mathrm{EP}_{4}>\mathrm{EP}_{2}$, and $\mathrm{EP}_{3}$ is the best one.

4.2. Comparative Analysis. In this part, the designed method is compared with four methods to show the superiority. Firstly, the research makes a comparison between our designed methods, IVIFWA and IVIFWG. For given IVIFWA operator, the calculating value is $S\left(\mathrm{EP}_{1}\right)=0.5821$, $S\left(\mathrm{EP}_{2}\right)=0.4709, S\left(\mathrm{EP}_{3}\right)=0.6324, S\left(\mathrm{EP}_{4}\right)=0.5013$, and $S\left(\mathrm{EP}_{5}\right)=0.5632$. Thus, the ranking order is $\mathrm{EP}_{3}>\mathrm{EP}_{1}>\mathrm{EP}_{5}>\mathrm{EP}_{4}>\mathrm{EP}_{2}$. For the given IVIFWG information operator, the order value is $S\left(\mathrm{EP}_{1}\right)=0.5633$, $S\left(\mathrm{EP}_{2}\right)=0.4543, S\left(\mathrm{EP}_{3}\right)=0.6288, S\left(\mathrm{EP}_{4}\right)=0.5087$, and $S\left(\mathrm{EP}_{5}\right)=0.5431$. So, the order is $\mathrm{EP}_{3}>\mathrm{EP}_{1}>$ $\mathrm{EP}_{5}>\mathrm{EP}_{4}>\mathrm{EP}_{2}$.

Furthermore, the designed method is compared with IVIF-MABAC [42]. The given overall value of each given alternative is as follows: $I_{1}=2.6576, \quad I_{2}=0.5324$, $I_{3}=4.0232, I_{4}=0.9855$, and $I_{5}=1.2577$. Therefore, the order of all given alternatives is $\mathrm{EP}_{3}>\mathrm{EP}_{1}>\mathrm{EP}_{5}>\mathrm{EP}_{4}>\mathrm{EP}_{2}$. Finally, our defined method is compared with IVIF-CODAS [43]. The total assessment score (AS) of each given alternative is as follows: $\mathrm{AS}_{1}=0.9253, \mathrm{AS}_{2}=-1.6588$, $\mathrm{AS}_{3}=1.6754, \mathrm{AS}_{4}=-1.1277$, and $\mathrm{AS}_{5}=0.6577$. Therefore, the order of alternatives is $\mathrm{EP}_{3}>\mathrm{EP}_{1}>\mathrm{EP}_{5}>\mathrm{EP}_{4}>\mathrm{EP}_{2}$. 
TABLE 11: $\operatorname{IVIFPIS}\left(\xi_{i}\right)$ and $\operatorname{IVIFNIS}\left(\xi_{i}\right)$ of every alternative.

\begin{tabular}{lcc}
\hline Alternatives & IVIFPIS $\left(\xi_{i}\right)$ & IVIFNIS $\left(\xi_{i}\right)$ \\
\hline $\mathrm{EP}_{1}$ & 0.5653 & 0.4146 \\
$\mathrm{EP}_{2}$ & 0.4789 & 0.9398 \\
$\mathrm{EP}_{3}$ & 0.8524 & 0.3746 \\
$\mathrm{EP}_{4}$ & 0.4856 & 0.7449 \\
$\mathrm{EP}_{5}$ & 0.6275 & 0.4866 \\
\hline
\end{tabular}

TABLE 12: IVIFRRD of each alternative from IFPIS.

\begin{tabular}{lccccc}
\hline Alternatives & $\mathrm{EP}_{1}$ & $\mathrm{EP}_{2}$ & $\mathrm{EP}_{3}$ & $\mathrm{EP}_{4}$ & \\
\hline $\operatorname{IVIFRRD}\left(\xi_{i}\right)$ & 0.5770 & 0.3375 & 0.6947 & 0.3946 & 0.5632 \\
\hline
\end{tabular}

TABLE 13: The obtained results of other methods.

\begin{tabular}{llcl}
\hline Models & \multicolumn{1}{c}{ Order } & The best choice & The worst choice \\
\hline IVIFWA operator [44] & $\mathrm{EP}_{3}>\mathrm{EP}_{1}>\mathrm{EP}_{5}>\mathrm{EP}_{4}>\mathrm{EP}_{2}$ & $\mathrm{EP}_{3}$ & $\mathrm{EP}_{2}$ \\
IVIFWG operator [13] & $\mathrm{EP}_{3}>\mathrm{EP}_{1}>\mathrm{EP}_{5}>\mathrm{EP}_{4}>\mathrm{EP}_{2}$ & $\mathrm{EP}_{2}$ \\
IVIF-MABAC method [42] & $\mathrm{EP}_{3}>\mathrm{EP}_{1}>\mathrm{EP}_{5}>\mathrm{EP}_{4}>\mathrm{EP}_{2}$ & $\mathrm{EP}_{3}$ & $\mathrm{EP}_{2}$ \\
IVIF-CODAS method [43] & $\mathrm{EP}_{3}>\mathrm{EP}_{1}>\mathrm{EP}_{5}>\mathrm{EP}_{4}>\mathrm{EP}_{2}$ & $\mathrm{EP}_{3}$ & $\mathrm{EP}_{2}$ \\
The designed method & $\mathrm{EP}_{3}>\mathrm{EP}_{1}>\mathrm{EP}_{5}>\mathrm{EP}_{4}>\mathrm{EP}_{2}$ & $\mathrm{EP}_{3}$ & $\mathrm{EP}_{2}$ \\
\hline
\end{tabular}

Ultimately, the results of these given methods are shown in Table 13.

From Table 13, it is evident that the most optimal choice is $\mathrm{EP}_{3}$, whereas the worst one is $\mathrm{EP}_{2}$. These methods could also cope with MAGDM from different angles. The IVIFWA and IVIFWG operator directly fuse given decision values. The IVIF-MABAC process emphasizes the risk preferences and interactive relationship among given criteria. The IVIFGRA emphasizes shape similarity degree between two given sequences. The IVIF-CODAS model often emphasizes the connection of practical Euclidean and concrete Hamming distance. However, compared with these previous four methods, the designed model may be more accurate, since it not only considers each alternative's closest degree to IVIFPIS but also considers each alternative's farthest degree from IVIFNIS.

\section{Discussion and Conclusion}

Although the final comparison revealed that the four methods achieved the same ranking result, illustrating the effectiveness of the proposed method, it is difficult to conclude that the results are always the same under all conditions. Their efficiencies may be different in practice. The proposed method calculates the IVIFPIS and IVIFNIS in replaced upper- and lower-approximation areas in the MABAC process [42] and is advantageous to e-commerce service evaluation. However, it does not focus on risk assessment, unlike MABAC, which may be vital for other management areas.

The simulation of the real world using models and algorithms has limitations. The proposed method estimated the average weight of all five experts in this research, and
IVIFWG could increase the weight of one expert through the weighted geometric mean [13]. Democratic and opinionleader models are considered by those two methods. In other situations, the weights of opinion leaders should not be increased individually. The assessment process should focus on similar or moderate dispersion comments. Further studies should consider more of such situations.

For agriculture e-commerce services, the inverted $U$ model is another knotty problem. High evaluation data of one attribute do not imply that the service is good in terms of that attribute (e.g., excessive enthusiasm). Algorithm improvement and research design avoiding may be two possible solutions for this problem that will be explored in the future.

This paper defines a useful method for this kind of issue, even the interaction between membership and nonmembership is considered in previous methods, such as Choquet-IVIF and interactive aggregation operators under IVIF [45-47], it builds the IVIF-GRA method for service quality evaluation of agricultural B2C e-commerce. After that, a numerical example gives evaluation to the service quality of agricultural B2C e-commerce. Furthermore, to check on the feasibility as well as availability of the new proposed method, some useful comparative analyses are also designed. In the near future, the defined models could also be extended to many more uncertain cases [48-51] and the designed algorithms could be employed to cope with many other real MAGDM-like evaluation issues and site selection [33,52]. A new possibility degree measure for interval-valued q-rung orthopair fuzzy sets in decision making can be explored for methodology innovation. 


\section{Data Availability}

The original dataset is included within the article (Tables 1-5). All 5 invited experts expressed their assessed information through four attributes.

\section{Conflicts of Interest}

The authors declare that there are no conflicts of interest regarding the publication of this article.

\section{Acknowledgments}

This research was funded by the Startup Foundation of Talents of Suqian University.

\section{References}

[1] D.-F. Li and S.-P. Wan, "Fuzzy heterogeneous multiattribute decision making method for outsourcing provider selection," Expert Systems with Applications, vol. 41, no. 6, pp. 3047-3059, 2014.

[2] H. Garg and Nancy, "Linguistic single-valued neutrosophic prioritized aggregation operators and their applications to multiple-attribute group decision-making," Journal of Ambient Intelligence and Humanized Computing, vol. 9, no. 6, pp. 1975-1997, 2018.

[3] M. Kracka, W. K. M. Brauers, and E. K. Zavadskas, "Ranking heating losses in a building by applying the MULTIMOORA," Inzinerine Ekonomika-Engineering Economics, vol. 21, pp. 352-359, 2010.

[4] L. A. Zadeh, "Fuzzy sets," Information and Control, vol. 8, no. 3, pp. 338-353, 1965.

[5] K. T. Atanassov, "Intuitionistic fuzzy sets," Fuzzy Sets and Systems, vol. 20, no. 1, pp. 87-96, 1986.

[6] P. Gupta, H. D. Arora, and P. Tiwari, "Generalized entropy for intuitionistic fuzzy sets," Malaysian Journal of Mathematical Sciences, vol. 10, pp. 209-220, 2016.

[7] Y. He, Z. He, and H. Huang, "Decision making with the generalized intuitionistic fuzzy power interaction averaging operators," Soft Computing, vol. 21, no. 5, pp. 1129-1144, 2017.

[8] S.-M. Chen, S.-H. Cheng, and T.-C. Lan, "Multicriteria decision making based on the TOPSIS method and similarity measures between intuitionistic fuzzy values," Information Sciences, vol. 367-368, pp. 279-295, 2016.

[9] F. Jin, Z. Ni, H. Chen, and Y. Li, "Approaches to group decision making with intuitionistic fuzzy preference relations based on multiplicative consistency," Knowledge-Based Systems, vol. 97, pp. 48-59, 2016.

[10] P. Liu, J. Liu, and S.-M. Chen, "Some intuitionistic fuzzy Dombi Bonferroni mean operators and their application to multi-attribute group decision making," Journal of the Operational Research Society, vol. 69, no. 1, pp. 1-24, 2018.

[11] R. X. Liang, S. S. He, J. Q. Wang, K. Chen, and L. Li, “An extended MABAC method for multi-criteria group decisionmaking problems based on correlative inputs of intuitionistic fuzzy information," Computational \& Applied Mathematics, vol. 38, p. 28, 2019.

[12] L. Zhang and Y. He, "Extensions of intuitionistic fuzzy geometric interaction operators and their application to cognitive microcredit origination," Cognitive Computation, vol. 11, no. 5, pp. 748-760, 2019.
[13] Z. Xu and R. R. Yager, "Some geometric aggregation operators based on intuitionistic fuzzy sets," International Journal of General Systems, vol. 35, no. 4, pp. 417-433, 2006.

[14] K. Krishankumar, R. Ravichandran, and A. B. Saeid, "A new extension to PROMETHEE under intuitionistic fuzzy environment for solving supplier selection problem with linguistic preferences," Applied Soft Computing, vol. 60, pp. 564-576, 2017.

[15] M. Li and C. Wu, "A distance model of intuitionistic fuzzy cross entropy to solve preference problem on alternatives," Mathematical Problems in Engineering, vol. 20169 pages, 2016.

[16] R. Joshi and S. Kumar, "An intuitionistic fuzzy information measure of order- (alpha, beta) with a new approach in supplier selection problems using an extended VIKOR method," Journal of Applied Mathematics and Computing, vol. 60, no. 1-2, pp. 27-50, 2019.

[17] P. Phochanikorn and C. Q. Tan, "A new extension to a multicriteria decision-making model for sustainable supplier selection under an intuitionistic fuzzy environment," Sustainability, vol. 11, p. 24, 2019.

[18] P. Li, J. Liu, S. F. Liu, X. Su, and J. Wu, "Grey target method for intuitionistic fuzzy decision making based on grey incidence analysis," Journal of Grey System, vol. 28, pp. 96-109, 2016.

[19] K. Kumar and H. Garg, "TOPSIS method based on the connection number of set pair analysis under interval-valued intuitionistic fuzzy set environment," Computational and Applied Mathematics, vol. 37, no. 2, pp. 1319-1329, 2018.

[20] T. He, G. Wei, J. Lu, J. Wu, C. Wei, and Y. Guo, “A novel EDAS based method for multiple attribute group decision making with pythagorean 2-tuple linguistic information," Technological and Economic Development of Economy, vol. 26, no. 6, pp. 1125-1138, 2020.

[21] J. L. Deng, "Introduction to grey system," The Journal of Grey System, vol. 1, pp. 1-24, 1989.

[22] Y. S. Tan, H. Chen, and S. Wu, "Evaluation and implementation of environmentally conscious product design by using AHP and grey relational analysis approaches," Ekoloji, vol. 28, pp. 857-864, 2019.

[23] A. Malek, S. Ebrahimnejad, and R. Tavakkoli-Moghaddam, "An improved hybrid grey relational analysis approach for green resilient supply chain Network assessment," Sustainability, vol. 9, 2017.

[24] M. Yazdani, C. Kahraman, P. Zarate, and S. C. Onar, "A fuzzy multi attribute decision framework with integration of QFD and grey relational analysis," Expert Systems with Applications, vol. 115, pp. 474-485, 2019.

[25] C. H. Chen, "A new multi-criteria assessment model combining GRA techniques with intuitionistic fuzzy entropybased TOPSIS method for sustainable building materials supplier selection," Sustainability, vol. 11, Article ID 2265, 2019.

[26] B. Zhu, L. Yuan, and S. Ye, "Examining the multi-timescales of European carbon market with grey relational analysis and empirical mode decomposition," Physica A: Statistical Mechanics and Its Applications, vol. 517, pp. 392-399, 2019.

[27] Z. P. Chiang, "Extended grey relational analysis for dependent criteria decision making problems," Journal of Grey System, vol. 23, pp. 273-280, 2011.

[28] O. Alptekin, N. Alptekin, and B. Sarac, "Evaluation of low carbon development of European union countries and Turkey using grey relational analysis," Tehnicki Vjesnik-Technical Gazette, vol. 25, pp. 1497-1505, 2018.

[29] C.-Y. Kung and K.-L. Wen, "Applying Grey Relational Analysis and Grey Decision-Making to evaluate the 
relationship between company attributes and its financial performance-A case study of venture capital enterprises in Taiwan," Decision Support Systems, vol. 43, no. 3, pp. 842-852, 2007.

[30] F. Lei, J. Lu, G. Wei, J. Wu, C. Wei, and Y. Guo, "GRA method for waste incineration plants location problem with probabilistic linguistic multiple attribute group decision making," Journal of Intelligent \& Fuzzy Systems, vol. 39, no. 3, pp. 2909-2920, 2020.

[31] G. Wei and G. Lan, "Grey relational analysis method for interval-valued intuitionistic fuzzy multiple attribute decision making," in Proceedings of the 2008 Fifth International Conference on Fuzzy Systems and Knowledge Discovery, pp. 291-295, Jinan, China, November 2008.

[32] H. Gao, M. Lu, and Y. Wei, "Dual hesitant bipolar fuzzy hamacher aggregation operators and their applications to multiple attribute decision making," Journal of Intelligent \& Fuzzy Systems, vol. 37, no. 4, pp. 5755-5766, 2019.

[33] Y.-L. Li, R. Wang, and K.-S. Chin, "New failure mode and effect analysis approach considering consensus under interval-valued intuitionistic fuzzy environment," Soft Computing, vol. 23, no. 22, pp. 11611-11626, 2019.

[34] S.-M. Chen and K.-Y. Fan, "Multiattribute decision making based on probability density functions and the variances and standard deviations of largest ranges of evaluating intervalvalued intuitionistic fuzzy values," Information Sciences, vol. 490, pp. 329-343, 2019.

[35] Z. S. Xu and J. Chen, "An overview of distance and similarity measures of intuitionistic Fuzzy Sets," International Journal of Uncertainty, Fuzziness and Knowledge-Based Systems, vol. 16, no. 4, pp. 529-555, 2008.

[36] L. Abdullah, N. Zulkifli, H. Liao, E. Herrera-Viedma, and A. Al-Barakati, "An interval-valued intuitionistic fuzzy DEMATEL method combined with Choquet integral for sustainable solid waste management," Engineering Applications of Artificial Intelligence, vol. 82, pp. 207-215, 2019.

[37] D. Kong, T. Chang, J. Pan et al., "A decision variable-based combinatorial optimization approach for interval-valued intuitionistic fuzzy MAGDM," Information Sciences, vol. 484, pp. 197-218, 2019.

[38] D. Diakoulaki, G. Mavrotas, and L. Papayannakis, "Determining objective weights in multiple criteria problems: the critic method," Computers \& Operations Research, vol. 22, no. 7, pp. 763-770, 1995.

[39] C. Gronroos, Service Marketing Management, Fudan University Press, Shanghai, China, 1998.

[40] A. Parasuramn, V. A. Zeithaml, and L. L. Berry, "A conceptual model of service quality and its implications for future research," Journal of Marketing, vol. 49, pp. 44-50, 1985.

[41] J. J. Cronin Jr. and S. A. Taylor, "Measuring service quality: a reexamination and extension," Journal of Marketing, vol. 56, no. 3, pp. 55-68, 1992.

[42] H.-C. Liu, J.-X. You, and C.-Y. Duan, "An integrated approach for failure mode and effect analysis under intervalvalued intuitionistic fuzzy environment," International Journal of Production Economics, vol. 207, pp. 163-172, 2019.

[43] E. Bolturk and C. Kahraman, "Interval-valued intuitionistic fuzzy CODAS method and its application to wave energy facility location selection problem," Journal of Intelligent \& Fuzzy Systems, vol. 35, no. 4, pp. 4865-4877, 2018.

[44] Z.-x. Su, G.-p. Xia, and M.-y. Chen, "Some induced intuitionistic fuzzy aggregation operators applied to multi-attribute group decision making," International Journal of General Systems, vol. 40, no. 8, pp. 805-835, 2011.
[45] G.. Harish, A.. Nikunj, and A. Tripathi, "Choquet integralbased information aggregation operators under the intervalvalued intuitionistic fuzzy set and its applications to decision making process," International Journal for Uncertainty Quantification, vol. 7, pp. 249-269, 2017.

[46] H. Garg, "A new generalized improved score function of interval-valued intuitionistic fuzzy sets and applications in expert systems," Applied Soft Computing, vol. 38, pp. 988-999, 2016.

[47] H. Garg, N. Agarwal, and A. Tripathi, "Some improved interactive aggregation operators under interval-valued intuitionistic fuzzy environment and its application to decision making process," Scientia Iranica, vol. 24, no. 5, pp. 2581-2604, 2017.

[48] G.-F. Yu, D.-F. Li, and W. Fei, "A novel method for heterogeneous multi-attribute group decision making with preference deviation," Computers \& Industrial Engineering, vol. 124, pp. 58-64, 2018.

[49] G. Wei, J. Wu, Y. Guo, J. Wang, and C. Wei, “An extended COPRAS model for multiple attribute group decision making based on single-valued neutrosophic 2-tuple linguistic environment," Technological and Economic Development of Economy, vol. 27, no. 2, pp. 353-368, 2021.

[50] P. Ren, Z. Xu, and H. Liao, "Intuitionistic multiplicative analytic hierarchy process in group decision making," Computers \& Industrial Engineering, vol. 101, pp. 513-524, 2016.

[51] H. Zhang, C. Takanashi, S. Si, G. Zhang, and L. Wang, "How does multimedia word of mouth influence consumer trust, usefulness, dissemination and gender," European J. of International Management, vol. 13, no. 6, pp. 785-809, 2019.

[52] E. K. Zavadskas, Z. Turskis, and V. Bagočius, "Multi-criteria selection of a deep-water port in the eastern baltic sea," Applied Soft Computing, vol. 26, pp. 180-192, 2015. 